\title{
HYPERTENSION AFTER RESECTION OF COARCTATION OF THE AORTA
}

\author{
BY \\ CARL J. INGOMAR AND ERIK TERSLEV \\ From the Department of Padiatrics, Rigshospital (Head: Professor Preben Plum) and the Dronning Louises Childrens \\ Hospital (Head: Professor Oluf Andersen), Copenhagen, Denmark
}

Received December 29, 1960

The arterial hypertension that is found in the upper limbs in cases of coarctation of the aorta disappears in the course of a longer or shorter period after the excision of the stenosis (cit. Karnell, 1959). In recent years it has, however, been observed that during the post-operative period the blood pressure may rise to above the pre-operative level, a phenomenon that has been termed by Lepere (1957) reactive or paradoxical hypertension. Reactive hypertension is understood to mean the occurrence, after an operation for coarctation of the aorta, of a systolic or diastolic blood pressure exceeding that observed before operation, provided that this has been productive of demonstrable pulsation in the femoral arteries. This hypertension can be a serious complication. Having been confronted with several patients who, following resection of a coarctation, have for this reason presented diagnostic and therapeutic problems, we have gone through the case histories of a number of children upon whom this operation has been performed, with special reference to the postoperative blood pressure levels. These findings are reported in this paper.

It is difficult to obtain any clear impression of the frequency of reactive hypertension. It is not mentioned by Karnell (1959) in his thesis on the blood pressure before and after operation for coarctation of the aorta. Lepere (1957) describes it as occurring in 45 out of 61 patients $(75 \%)$, and Sealy et al. (1957) in 21 out of $31(68 \%)$. It is not possible to ascertain the sex distribution in Lepere's material, but in Sealy's group the male: female ratio was 4:1. As far as can be determined from this material, the age at operation does not appear to bear any relation to the occurrence of the complication. Of Lepere's material, there was a raised systolic in 50 per cent of the patients with reactive hypertension and a raised diastolic pressure in 92 per cent, the average increases being 17 and $16 \mathrm{~mm} . \mathrm{Hg}$ respectively. By one month after operation the blood pressure was in all cases below the pre-operative level. Sealy et al. (1957) divide cases of reactive hypertension into one group showing "immediate hypertension", occurring within 12 hours of operation, lasting about 36 hours, and comprising mainly a rise of systolic pressure; and a second group showing "delayed hypertension", where the rise in pressure begins about 48 hours after operation, lasts from 6-8 days, and consists mainly of an elevation of the diastolic component. Only the latter group, that is the patients with "delayed hypertension", is of any great clinical importance.

Nothing definite is known of the atiology of reactive hypertension. Goodall et al. (cited by Sealy et al., 1957) have demonstrated a greater secretion of adrenalin during the post-operative period in patients operated upon for coarctation of the aorta than in those who had undergone other operations, and this could suggest the influence of humoral factors. It is known from experimental physiology that unilateral renal ischæmia can give rise to hypertension, and it is therefore easy to imagine that renal ischæmia could be the cause of reactive hypertension, but nothing of this nature has as yet been proved. Byrom and Dodson (1948) have, however, in experiments on the rat, caused necrotic changes in the renal vessels by means of a local rise of pressure in the common carotid artery, produced by the infusion of Ringer's solution. 
The course after operation for a coarctation of the aorta may further be complicated by the occurrence of abdominal attacks, which may arise in conjunction with reactive hypertension or as a separate entity (Grow et al., 1956). These attacks are characterized by the occurrence of abdominal pain, often accompanied by signs of ileus necessitating laparotomy, and occur a variable number of days after operation. They have been described by a number of authors, most recently by Gammelgaard and Friis-Hansen (1960), to whose paper reference should be made. Outside the intestinal canal other abdominal organs may be affected: hepatic and mesenteric arteries (Lober and Lillehei, 1954), vessels in the bladder, adrenals, kidneys, and renal pelvices (Perez-Alvarez and Oudkerk, 1955), diaphragm, spleen, stomach, pancreas, liver, and abdominal muscles (Benson and Sealy, 1956; Sealy, 1953). Several of those authors who have described post-operative abdominal attacks mention also reactive hypertension (Hurt and Hanbury, 1957; and Downing et al., 1958).

The microscopic changes found in the vessels of organs removed during these abdominal operations consist predominantly of necrosis of the arterial walls with neutrophil infiltration. In occasional cases it has been possible to demonstrate only thrombosis in the mesenteric vessels. Several authors (Benson and Sealy, 1956; Lober and Lillehei, 1954; Perez-Alvarez and Oudkerk, 1955; and Ring and Lewis, 1956) have found that the essential lesion is damage to the intima of the artery, probably due to overstretching of the intima followed by necrosis of the elastic lamina and the media. This, in the course of time, leads to the development of ischæmic changes in the corresponding organs. However, Reid and Dallachy (1958) believe that on account of the sudden rise in the blood pressure a protective arterial contraction occurs in order to shield the intima, and that the consequent reduction in blood flow leads to thrombosis in the vessels concerned.

Of the complications that seem directly attributable to reactive hypertension, two of those described by Lepere (1957) must be mentioned. In one case there was rupture of an aneurysm in a homotransplant, and in the other a subarachnoid hæmorrhage. Both events occurred in relation to reactive hypertension, and both resulted in the death of the patient.

The management of reactive hypertension has been widely disputed. Lepere (1957) found no difference in the frequency of the occurrence of reactive hypertension in patients operated upon in hypotensive anæsthesia, as compared with a group operated upon without this. Nor do we know the answer to the question whether the condition should be treated in its early stages, and if so by what means. Downing et al. (1958) think that reactive hypertension is a compensatory mechanism for ensuring the blood supply to the organs, and Reid and Dallachy (1958) consider that the arterial contraction acts as a protection to the capillaries, and that if such be the case then hypotensive therapy can be directly injurious. If, on the other hand, one allows the arterial contraction to continue, there is an increased risk of thrombosis. In two of the patients described by Sealy $e t$ al. (1957) antihypertensive therapy had a beneficial effect on the abdominal pain, but the treatment itself is not without risk. Lepere (1957), for example, quotes a case where it resulted in cortical necrosis of the kidney, with anuria and irreversible shock leading to death.

\section{Present Material}

Our material consists of those children with coarctation of the aorta who have been treated since 1950 in the Department of Pædiatrics, Rigshospital (operated upon by the staff of Surgical Units D and R) and since 1955 in the Dronning Louises Childrens Hospital. The two departments have co-operated in such a way that the older children have been admitted to Rigshospital, and the younger to the Childrens Hospital. In all, there have been 33 children, whose age distribution is shown in Fig. 1. Of these 33 (comprising 11 girls and 22 boys) there have been 5 ( 2 boys and 3 girls) in whom there was a post-operative rise in blood pressure to over the pre-operative level, that is an incidence of approximately 15 per cent.

From the practical view point it was most frequently possible to measure only the systolic blood pressure (by palpation). A measurement was made every 15 minutes during the first 48 hours after operation, and thereafter the frequency depended upon the condition of the patient.

Fig. 2 shows the range of blood pressures before and after operation of all those patients who 


\section{NUMBER}

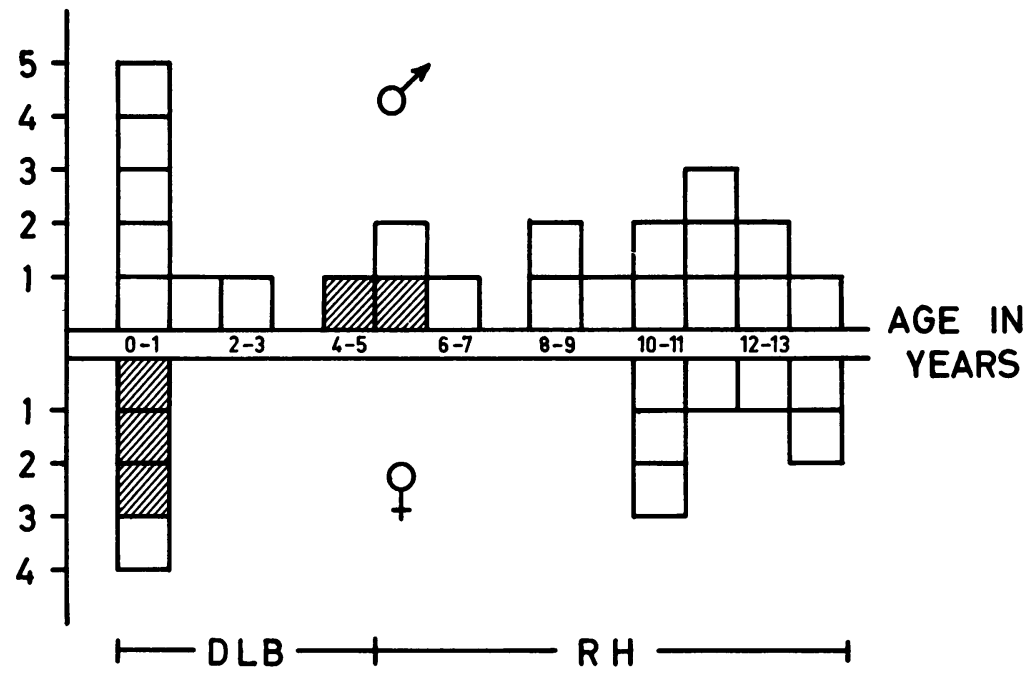

FIG. 1.-Age distribution of the patients. Each square represents one patient, and the cross-hatching indicates the occurrence of reactive hypertension.

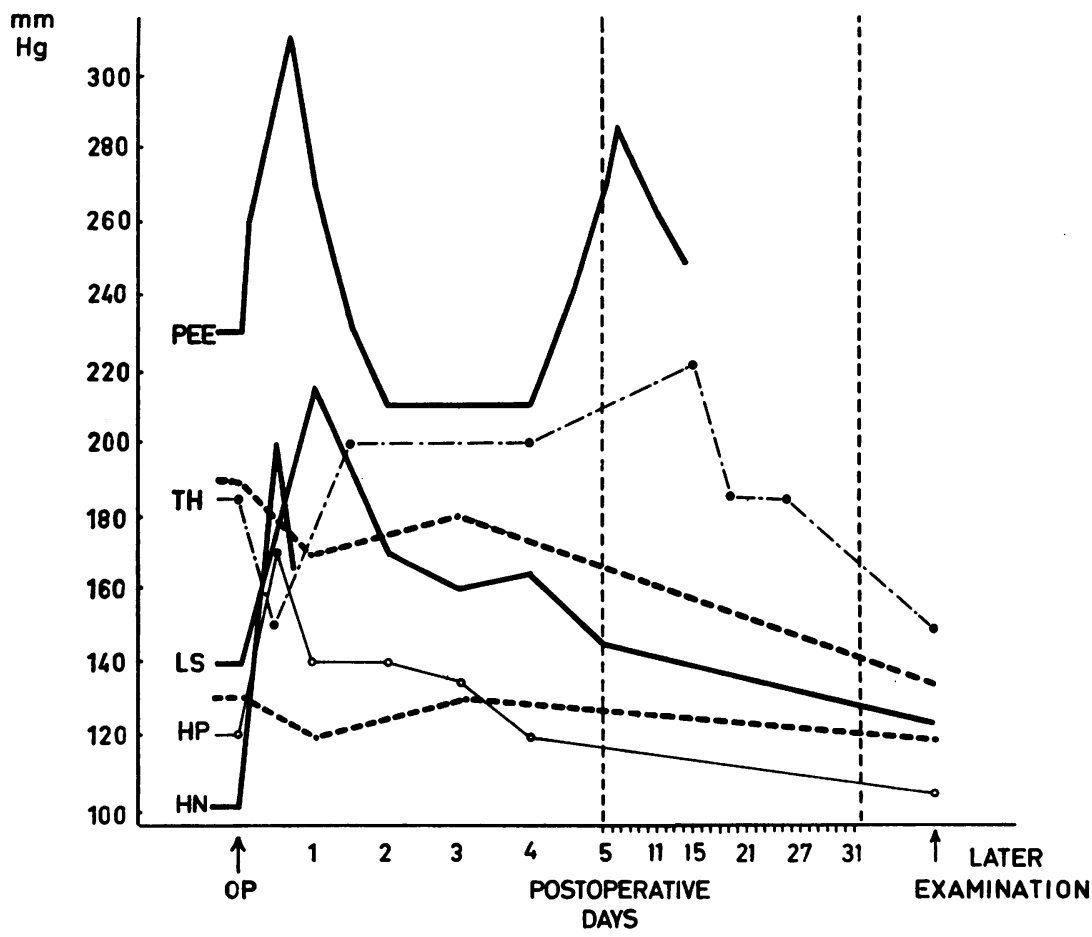

Fig. 2.-Post-operative course of the blood pressure. The two heavy dotted lines enclose the range of the post-operative blood pressures of the 19 patients over 6 years of age, in none of whom reactive hypertension occurred (see text). The blood pressures of the 5 patients with post-operative reactive hypertension are also shown, and marked individually with initials. 
were over 6 years of age at the time of operation, in none of whom reactive hypertension occurred. When these blood pressures are plotted on a graph it is found that the curves follow a similar pattern, consisting of a moderate fall on the first post-operative day, followed by a slight rise usually lasting for about two days, and never reaching the pre-operative level. Thereafter there is a gradual fall to normal values. The starting point, i.e. the pre-operative blood pressure, naturally varies from case to case, but the curves are all similar. On the graph the upper level of the range represents the values in the patient with the highest, and the lower level those in the patient with the lowest, pre-operative blood pressure (the other curves run parallel to and between these). The range thus represents the usual course of the blood pressure following operation for coarctation of the aorta. On the same graph the blood pressures before and after operation of the 5 children with reactive hypertension are also plotted. It can be seen that with a single exception the blood pressure rose to above that observed pre-operatively within 24 hours. In the one exception the rise first became obvious on the second day, but in all the rise was considerable.

\section{CASE Histories}

Case 1. H. P. (1210/59). A 2-month-old girl suffered from a cyanotic attack, with cough and dyspnœa two weeks before admission. On examination there was a præcordial murmur, signs of heart failure, and impalpable femoral pulses. The blood pressure in the upper limbs (measured by the flush method) was 120 and in the lower limbs $60 \mathrm{~mm}$. Hg. Operation for excision of the coarctation was performed. There was hypoplasia of a $1.5 \mathrm{~cm}$. long section of the aorta between the origins of the subclavian and common carotid arteries. While the area was being dissected out cardiac arrest occurred, but the heart action was restored after the intracardial injection of calcium chloride and the institution of cardiac massage. The remainder of the operation, consisting of excision and end-to-end anastomosis, was uncomplicated. The coarctation was found to have been nearly complete. The aorta was occluded for approximately 20 minutes. During operation the blood pressure lay in the region of 150/120, and at its conclusion was 160/110. After a fall to $100-110 \mathrm{~mm}$. in the first hour, it rose gradually over the course of the next six hours to $200 \mathrm{~mm}$., falling again in a further six hours to $130 \mathrm{~mm}$. During the rise in pressure some twitching of the limbs was observed. The later course was uncomplicated, the blood pressure lying in the region of $130 \mathrm{~mm}$. At follow-up, 9 months later, the pressure had fallen to $100 \mathrm{~mm}$.

Case 2. H. N. (698/60). A 3-month-old girl, with Bonnevie-Ullrich-Turner syndrome, had not thriven after the age of 6 weeks and had been subject to cyanotic attacks. Examination showed a child with obvious signs of cardiac disease and in early failure. There was no palpable pulsation in the femoral arteries. The blood pressure in the upper limbs was $112 \mathrm{~mm}$. (by palpation) and in the lower $86 \mathrm{~mm}$. At operation, there was a large coarctation with a lumen of $0.5 \mathrm{~mm}$. During dissection of the area cardiac arrest occurred, but the heart action was restored after the intracardiac injection of calcium chloride. The remainder of the operation proceeded without complication, the aorta being occluded for 35 minutes. The blood pressure during operation was $100 / 80$, and at its conclusion $80 / 70$. The post-operative condition of the patient was poor, the blood pressure rising to $185 \mathrm{~mm}$. (by palpation). The child was pale above the level of the umbilicus and hyperæmic below it, the skin being universally cold. The pressure gradually rose to $200 \mathrm{~mm}$. in the next five hours, and then fell to $180 \mathrm{~mm}$. in the following four hours. Two hours later it had fallen to $160 \mathrm{~mm}$., when death suddenly occurred. There had been a good diuresis with macroscopic hæmaturia. Necropsy revealed endocardial fibro-elastosis (the presence of which had been suspected from an angiocardiogram), hypoplasia of the adrenal glands, arcuate kidney with double pelvis and ureter, and bilateral ovarian agenesis. Microscopy of the kidneys, spleen, liver, adrenals, pancreas, jejunum, superior mesenteric artery, and abdominal musculature showed these organs to be of essentially normal structure.

Case 3. L. S. (1472/59). A $2 \frac{1}{2}$-month-old girl had shown poor progress from birth and suffered from dyspnœic attacks. Examination revealed an ill child, in heart failure. The blood pressure in the upper limbs was 130-155 mm., and in the lower limbs $50-60 \mathrm{~mm}$., by palpation. After treatment with digitalis, operation for the excision of the coarctation, which was found to be of the adult type, and ligation of a persistent ductus was undertaken. During the excision cardiac arrest occurred, the action being restored after intracardiac injection of calcium chloride, defibrillation, and cardiac massage. The remainder of the operation proceeded without complication, there being no signs of cerebral damage evident at its conclusion. During operation the blood pressure was $140 / 110$, and at its close the pressure was 140/100. The aorta was occluded for 45 minutes. In the first 24 hours after operation the pressure rose to $180-190 \mathrm{~mm}$., and in the second day to $220-210 \mathrm{~mm}$. There was good pulsation in the femoral arteries, but the general condition of the child was poor, with slow twitching of the left limbs and the face, the abdomen being distended with a large amount of air. The condition remained unaltered during the first 48 hours, with continued spasms and unreactive pupils. On the supposition that these were due to hypertensive encephalopathy, an intravenous infusion of trimetaphan camphorsulphonate (arfonad) was set up, and by means of this the blood pressure was reduced to $150 \mathrm{~mm}$., but without any improvement in the clinical picture. As the serum 
electrolytes were found at this time to be at the lower limit of normal (serum potassium, $4 \cdot 1 \mathrm{mEq} . / 1$.; serum sodium, $135 \mathrm{mEq}$./1.; serum chloride, $85 \mathrm{mEq}$./1.; and serum bicarbonate $22.5 \mathrm{M}$.mol.) water intoxication was considered a possibility, and an attempt was therefore made to improve the condition by the intravenous infusion of $20 \mathrm{ml} .25$ per cent saccharose, without any obvious effect. However, after a further two days the condition gradually improved, and by the fourth day the blood pressure was stable at $140 \mathrm{~mm}$. At followup, 4 months later, the child was clinically normal, but the blood pressure was still $125 / 86 \mathrm{~mm}$.

Case 4. T. H. 753/60 (previously described in detail by Gammelgaard and Friis-Hansen, 1960). A 4-year-old boy had not exhibited signs of heart disease apart from some difficulty in walking. The blood pressure in the upper limbs was found to be raised $(185 / 110 \mathrm{~mm}$.) and there was no palpable femoral pulsation. Operation for the excision of a coarctation of the aorta proceeded uneventfully, the aorta being occluded for 35 minutes. The blood pressure at the conclusion was $170 \mathrm{~mm}$. by palpation. During the first 24 hours after operation the pressure lay at about 170-150, climbing on the second day to $190-200 \mathrm{~mm}$. There was good pulsation in the femoral arteries. For further details of the blood pressure see Fig. 2. The post-operative course was complicated by a transient albuminuria, without signs of urinary tract infection, which began on the second day, and further by a picture resembling ileus, which necessitated laparotomy on the 17th day. Necrosis of a greater part of the ileum was found, and $70 \mathrm{~cm}$. of ileum was resected. Histological examination of the specimen revealed a severe acute inflammatory process and eosinophillia, particularly around the vessel walls, which were in places more or less degenerated. One week later further laparotomy became necessary, owing to rupture of the wound, and an ileococostomy was formed. This was closed about two months later, after which the further course was uncomplicated. At follow-up, 6 months later, the cardiac condition was satisfactory, but steatorrheoa was present, probably as a result of the extensive removal of small intestine.

Case 5. P. E. E. (371/55). A boy, aged 4, had swelling of the left leg but no cardiac symptoms. Examination revealed a soft systolic murmur over the præcordium, maximal at the apex and transmitted into the back and audible along the spine, normal radial pulses, and weak pulsation in the abdominal aorta and right femoral artery: pulsation was not felt in the left femoral artery. There was slight œdema of the left leg. Palpatory blood pressure in the upper limbs was 200-230, in the lower limbs $140 \mathrm{~mm}$. No cause for the œdema was discovered, but the diagnosis of coarctation was made, operation not being considered indicated at this time. The patient was readmitted two years later, with a history of increasing swelling of the left leg since discharge, the swelling now reaching the buttock. There was, in addition, slight functional dyspnœa. His condition was unchanged apart from the now considerable œdema of the left leg. The blood pressure in the upper limbs was $230 / 130$ and in the lower $145 / 110$. Aortography visualized a coarctation: the pelvic and femoral arteries on the left side were slightly larger and more ramified than those on the right, and the veins were normal. At operation, no typical coarctation was seen, but immediately below the ligamentum arteriosum the aorta was found to be narrowed to one-third of its diameter above that level, and the width did not return to normal until on a level with the origin of the third intercostal artery. The narrow portion was excised, and found to have a lumen of 1-2 mm. The aorta was occluded for 35-40 minutes. Histological examination revealed hyalinization of the intima of the aortic wall. The blood pressure immediately after operation was $260 \mathrm{~mm}$.: this rose during the first 24 hours to $310 \mathrm{~mm}$., falling thereafter to $210 \mathrm{~mm}$. on the fourth day. It again rose slowly, and by the ninth day was $260 \mathrm{~mm}$. In the course of the first days the diuresis fell slowly and there was a simultaneous rise in the blood urea (3rd day; diuresis, $50 \mathrm{ml}$.; blood urea, $158 \mathrm{mg}$. per $100 \mathrm{ml}$.: 4th day; diuresis, $30 \mathrm{ml}$., serum potassium, $7.8 \mathrm{mEq} . / 1$.). This was thought to indicate the presence of tubular interstitial nephritis, occurring as a sequel to renal ischæmia under operation. A caval drip was set up, and 50 per cent glucose infused. The diuresis rose slightly next day, and the serum potassium fell, but thereafter the diuresis again dropped and the blood urea increased, being $373 \mathrm{mg}$. per $100 \mathrm{ml}$. on the seventh day. Twitching of the facial musculature was observed, and the chest films showed X-ray changes typical of a uræmic lung. The patient was therefore transferred to the Dialysis Unit, Kommune Hospital, Copenhagen. Here, after a period of observation, there was thought to be insufficient indication for use of the artificial kidney, and the patient was transferred back to Rigshospital, where on the fourteenth day a further thoracotomy was performed. This revealed a thrombosis at the site of the previous anastomosis, and it was found necessary to remove a further $5 \mathrm{~cm}$. of aorta, until a region having normal intima was reached. A piece of adult carotid artery was transplanted, but the patient died about twelve hours after operation.

Necropsy. Apart from the changes in the aorta noted at operation there was much hypertrophy of the left ventricle. There were signs of an old infarct in the right kidney, and of recent infarcts in the left kidney and spleen. Microscopical examination revealed no abnormality of the right kidney, but the left kidney showed extensive infarction of both cortex and medulla. In the periphery of the area of infarction there was a region of hyperæmia and hæmorrhage. The area between infarcts was normal.

Microscopical Examination of the Brain (Erna Christensen). The arterioles in the leptomeninges and brain substance show considerable hyalinization with thickening of the walls, but there are no thromboses despite areas of narrowing of the lumen. The arteries are normal. Some of the cortical ganglion cells show anoxic changes of varying degree, being swollen, chromophobic or atrophic. Diagnosis: cerebral arteriosclerosis (hypertensive encephalopathy). 
DISCUSSION

In our case material of 33 patients, whose sex distribution corresponds to that usually found in coarctation of the aorta, i.e. male: female 2:1 (Wood, 1956), there were 5 in whom reactive hypertension occurred, an incidence of 15 per cent. All 5 were under six years of age and, indeed, 3 were less than one year old. Thus age does appear to play some part in the occurrence of reactive hypertension, perhaps in the fact that in the younger patients there has been insufficient time for the development of a collateral circulation capable of functioning during operative occlusion of the aorta, and thereby preventing ischæmia of the abdominal organs, particularly the kidneys.

In 26 of the patients included in this material it was possible to ascertain the length of time for which the aorta was occluded at operation. This varied from 20-70 minutes, with an average of 37 minutes, but it has not been possible to establish any relationship between the period of occlusion and the occurrence of reactive hypertension (Fig. 3). The three youngest patients were realized

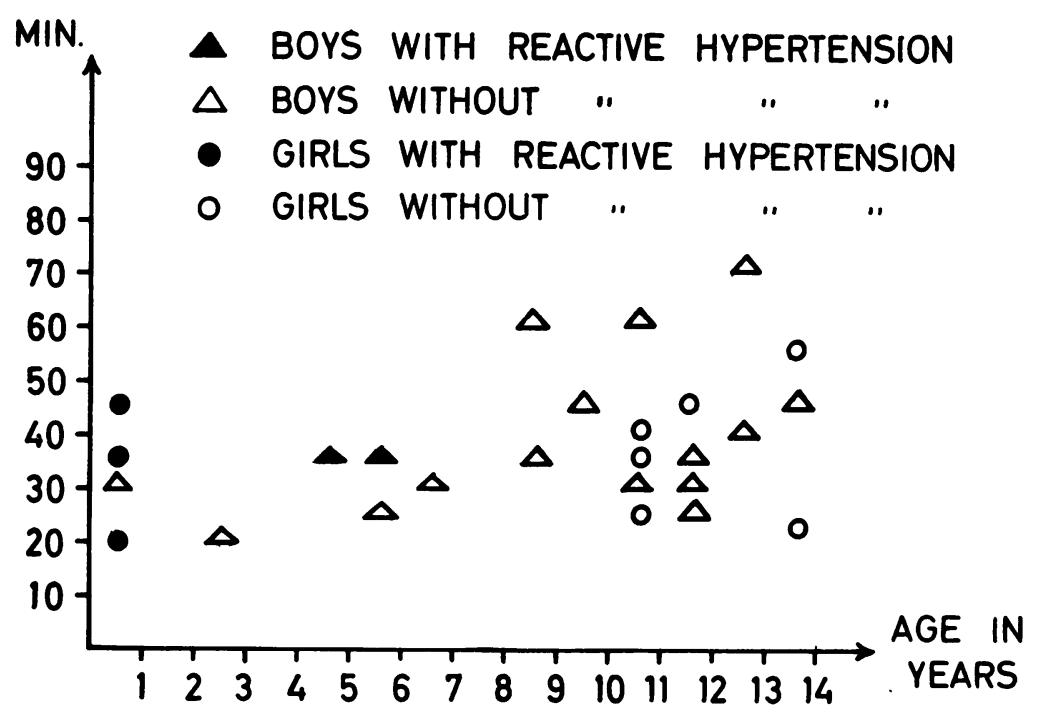

Fig. 3.-Number of minutes for which the aorta was occluded under operation in relation to the age of the patient in 26 cases (no information on this point was obtainable in the remaining 7 cases).

to be poor operative risks, but operation was considered essential in view of the degree of heart failure. In all three, cardiac arrest occurred during operation, and renal anoxia is perhaps therefore the most probable cause of the post-operative reactive hypertension.

In Case 2 there was polyuria and hæmaturia in the post-operative period, but no signs of renal damage were visualized at necropsy. This, however, does not exclude the possibility that there were, at some period, vascular changes in the kidneys sufficient to produce reactive hypertension. In Case 4 there was post-operative albuminuria and so, here also, there is the possibility that renal vascular changes were the cause of the reactive hypertension; the vascular changes found in the gangrenous intestine serving to strengthen this view. In Case 5 we think that the vascular changes in the kidney (or kidneys?) were due to ischæmia occurring during operation, probably when the clamps were removed from the aorta, when the sudden rise in the pressure of the blood arriving in the region of the kidneys resulted either in primary capillary damage or else in a primary contraction of the arterioles, changes that were followed by a secondary thrombosis; and that these changes were the reason for the reactive hypertension. In Case 3 we have been unable to demonstrate any urinary tract changes. The water intoxication might be considered as a possible causative factor, but the patient did not appear to benefit from treatment designed to combat this. In Case 1 we have no explanation for the reactive hypertension, except the possible occurrence of renal anoxia. 
Post-operative reactive hypertension is of no little significance, especially in very young patients, and we think that it was an important contributary cause of death in Case 2. The sudden rise in the blood pressure could have put such a severe strain on the heart, already affected by fibro-elastosis, that cardiac arrest occurred.

With reference to the prevention of post-operative reactive hypertension it seems probable that the period of occlusion of the aorta, especially in small children, should be reduced to the minimum, although we have been unable to demonstrate this in the present material. Probably, however, it is even more important that the release of the aortic clamps after anastomosis should be much slower than is at present considered necessary, in order to avoid any sudden rise in pressure in those regions of vascular supply (particularly the kidneys, spleen, and intestine) that have previously been subject to hypotension. There is no evidence that prophylactic hypotensive therapy is of any value.

Finally we believe that, in order to diagnose reactive hypertension, it is important to institute in the post-operative period, in addition to a control of the blood pressure, a system of frequent examination of the urine for erythrocytes and albumen (to detect vascular changes in the kidneys), the peripheral blood for leucocytosis (reaction to infarction), and electrolyte balance (water intoxication), and the freces for blood (intestinal infarction).

\section{SUMMARY}

An account is given of the post-operative course of the blood pressure in 33 children (aged 2 months-13 years) operated upon for coarctation of the aorta. Five of these $(15 \%)$ developed reactive hypertension, defined as a post-operative rise in blood pressure to above the pre-operative level. In one of these patients the reactive hypertension was presumably of renal origin, and in two others urinary symptoms pointed to a similar causation. The ætiology and pathogenesis are discussed, and from the prophylactic viewpoint it is recommended that the release of the aortic clamps after anastomosis be very slow.

\section{REFERENCES}

Benson, W. R., and Sealy, W. C. (1956). Lab. Invest., 5, 359.

Byrom, F. B., and Dodson, L. F. (1948). J. Path. Bact., 60, 357.

Downing, D. F., Grotzinger, P. J., and Weller, R. W. (1958). J. Dis. Child., 96, 711.

Gammelgaard, A., and Friis-Hansen, B. (1960). Acta chir. scandinav., 119, 361.

Grow, J. B., Demong, C. V., and Rundles, W. R. (1956). Ann. Surg., 22, 1168.

Hurt, R. L., and Hanbury, W. J. (1957). Thorax, 12, 258.

Karnell, J. (1959). Opusc. med. Suppl., 1.

Lepere, R. L. (1957). Guy's Hosp. Rep., 106, 128.

Lober, P. H., and Lillehei, C. W. (1954). Surgery, 35, 950.

Perez-Alvarez, J. J., and Oudkerk, S. (1955). Surgery, 37, 833.

Reid, H. C., and Dallachy, R. (1958). Brit. J. Surg., 45, 625.

Ring, D. M., and Lewis, F. J. (1956). J. thoracic Surg., 31, 718.

Sealy, W. C. (1953). Surg. Gynec. Obst., 97, 301.

, Harris, J. S., Young, W. G., Callaway, H. A., and Durham, N. C. (1957). Surgery, $42,135$.

Wood, P. (1956). Diseases of the Heart and Circulation. Eyre \& Spottiswoode, London. 